\title{
Characteristic Analysis of Mobility using Heterogeneous Network
}

\author{
Pulak Singh \\ Student, M.Tech. Scholar \\ Electronics \& Communication \\ Department Shepherd School of \\ Engineering \& Technology, \\ Sam Higginbottom Institute of \\ Agriculture, Technology \& \\ Sciences Allahabad, India.
}

\author{
Mayur Kumar \\ Asst. Professor \\ Electronics \& Communication \\ Department Shepherd School of \\ Engineering \& Technology, \\ Sam Higginbottom Institute of \\ Agriculture, Technology \& \\ Sciences Allahabad, India.
}

\author{
A.K.Jaiswal \\ Professor \& Head of the \\ Department, \\ Electronics \& Communication \\ Department Shepherd School of \\ Engineering \& Technology, \\ Sam Higginbottom Institute of \\ Agriculture, Technology \& \\ Sciences Allahabad, India.
}

\begin{abstract}
In present days, with increasing technologies in mobility management, the next generation client can access information from anywhere, anytime and whenever they want. To design such type of environment client can integrate various types of networks by using different engineering technologies. In this paper, the heterogeneous network in which WLAN (Wireless Local Area Network) and WiMAX networks are integrated with flag mobility and the QOS (Quality of Service) parameter is measured and analyzed in terms of throughput, average end to end delay and jitter by using QualNet 6.1 simulator.
\end{abstract}

\section{Keywords}

Heterogeneous Network, Mobility Management, Seamless Mobility, QualNet 6.1

\section{INTRODUCTION}

Now days, the demand of wireless networks are increasing day by day to meet the requirement of users in various field such as high speed processors. In the present era the interacting technologies are escalating continuously such as Wi-Fi and WLAN technologies. The researchers yielded their interest in the different networking field such as peer to peer network, cellular wireless, file server, World Wide Web and grid computing system. Seamless mobility in distributed network provides the best QOS to the clients. Seamless mobility is important to accommodate the demand of seamless mobility in wireless network. The seamless data transfer in wireless network is of great concern. The problems are due to the hidden terminal collision and RTS/CTS in wireless network incompleteness protocol.

These constraints arise as a result in seamless and secure networking:-

- Due to time delay in inter system roaming.

- These disturbances are eliminated by the fresh network connection.

- With the accumulation of various different modern technologies to interact with the environment increases the complexities.

The work related with the characteristic analysis of mobility using heterogeneous network is described in section 2, the simulation setup is demonstrated in section 3, results are analyzed in section 4 , and final conclusion in section 5 .

\section{RELATED WORK}

As well as increasing the number of mobile users, the area in the wireless communication technologies arises with the research activities. A new emerging technology proposed by reference [1] that provides a framework for media independent handover. The 'always best connected' that was allowed person connectivity to application using the devices described by reference [2]. Host based and mobility based management approach is described in reference [3]. Reference [4] also proposed 'host based network'. The current serving network is handoff to its target network to maintain the continuity of service without disconnection by reference [5]. In reference [6] proposed that WiMAX uses the reacting protocol and they provide point to point communication. In reference [7] showed that the merging of two technology give better result.

\section{SIMULATION SETUP}

This research has used QualNet simulator to simulate an integration of WIFI and WLAN network. To design a scenario QualNet simulator provide a graphical user interface. The heterogeneous network of WIFI and WLAN provides seamless mobility with vertical handover.

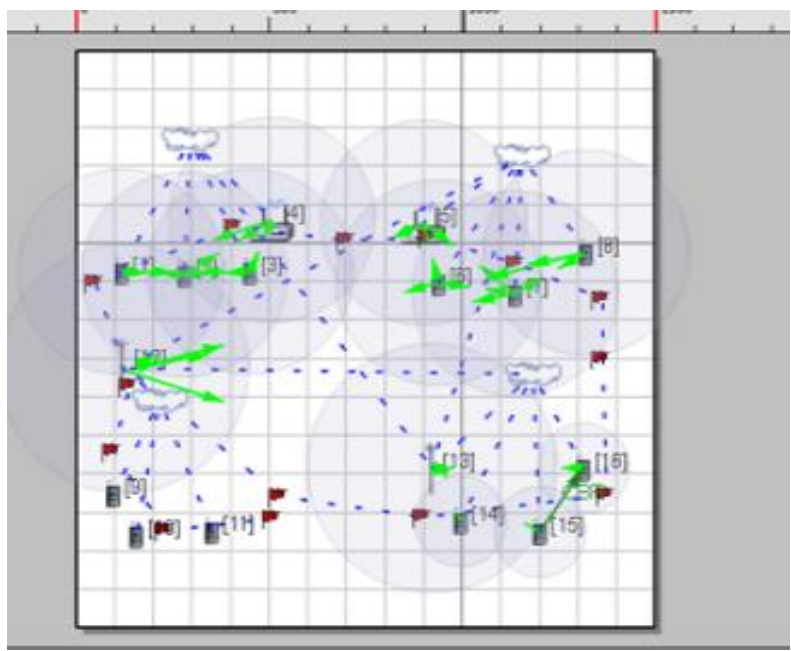

Fig 1: GUI Architecture of the double heterogeneous network 
Fig 1 described that double heterogeneous architecture in which two networks are WIFI and WLAN every network is connected with each other. In WLAN 802.11 network make any node as an access point and WIFI 802.16 make any node as a base station.

In this scenario we use the 16 number of nodes with the 1000 packets were transmitted. The Ad-hoc On Demand distance Vector (AODV) protocol connection with data traffic of constant bit rate was employed. Flag mobility is applied in this scenario which provide seamless mobility with no packet loss.

\begin{tabular}{|l|l|}
\hline \multicolumn{1}{|c|}{ Parameters } & \multicolumn{1}{c|}{ Values } \\
\hline Channel type & Wireless Channel \\
\hline No Of Nodes & 16 \\
\hline Traffic Source & CBR \\
\hline Routing Protocol & AODV \\
\hline Antenna & Omni directional \\
\hline Simulation Time & 1030 \\
\hline Channel Frequency & $2.4,2.42,2.46,2.48$ \\
& \\
\hline
\end{tabular}

Table 1. Parameter of Simulation

\section{SIMULATION RESULTS AND ANALYSIS}

The QualNet 6.1 simulates a network with graphical user interface. Using this simulator analyze the parametric values total received message, throughput, end to end delay, jitter.

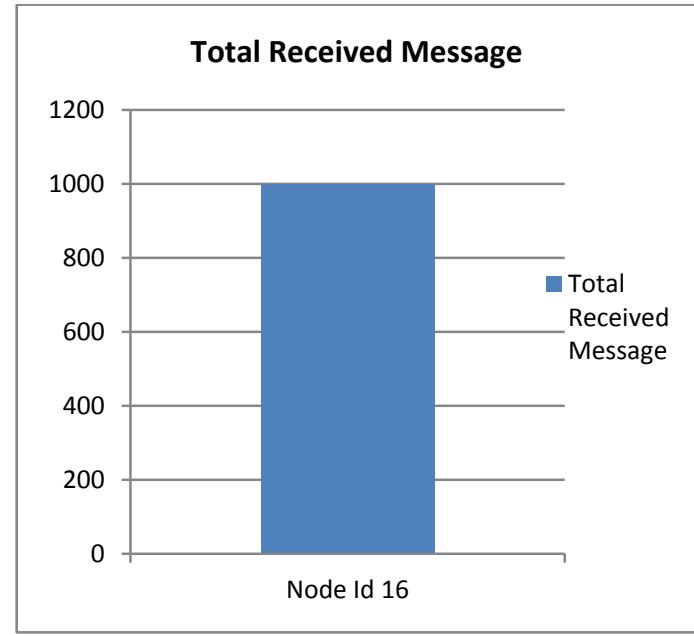

Fig 2: Total Received Message

Fig (2) shows that transmission and reception of packets from source node to destination node. From fig it is clear that the number of packet sends by source node is equal to the number of packet received by destination node with constant bit rate. It means that there is no data loss in the network with roaming connectivity.

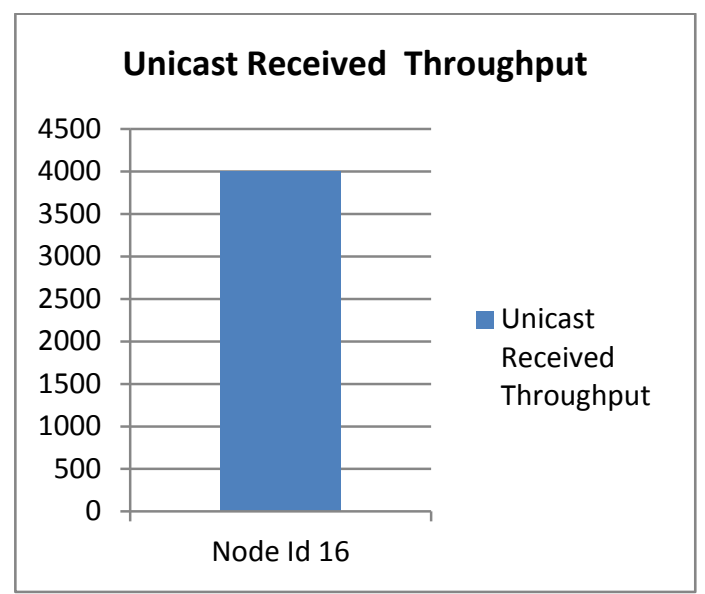

Fig 3: Unicast Received Throughput

Fig (3) Unicast received throughput, the data is evaluated 4000 bits/second.

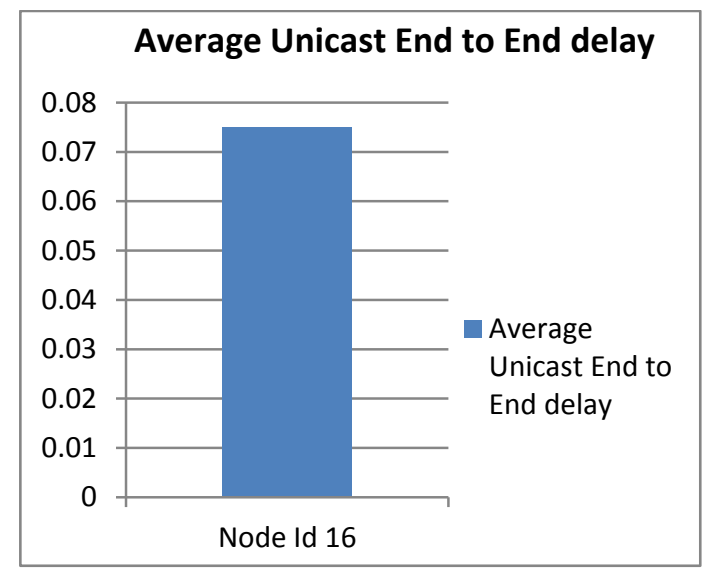

Fig 4: Average Unicast End to End Delay

Fig (4) Average Unicast End to End Delay shows the value of delay in 0.07 seconds.

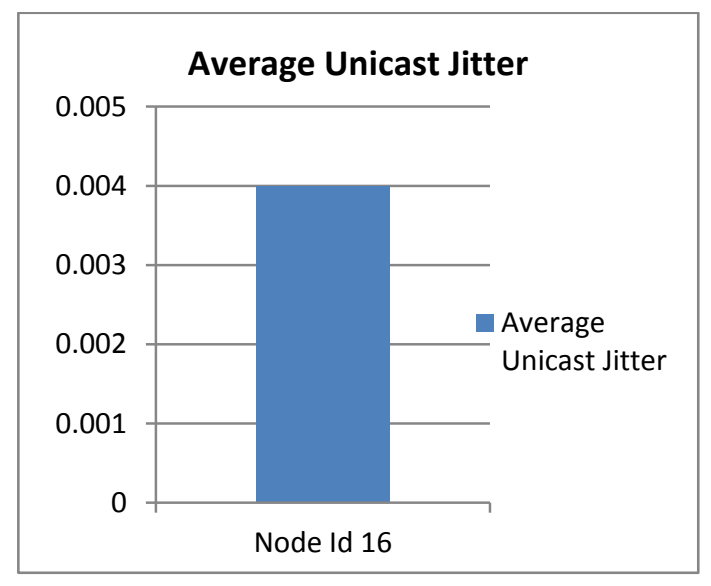

Fig 5: Average Unicast Jitter

Fig (5) Average Unicast Jitter the value is 0.004 seconds. 
Simulation results shows that the total packet received are equal to the number of packets sent that means there is no loss in packet and therefore this network provides seamless mobility. In this network sender transmits 1000 packets and receiver also receives 1000 packets.

\section{CONCLUSION \& FUTURE SCOPE}

To create a wireless communication network in a wide area, provide a seamless mobility without a disconnection of a network. The integration of WIFI and WLAN network provide better quality of service in the network. There is no packet loss and it reduces the delay in wireless network.

Wi-Fi in comparison to WiMAX is superior response of a wireless network. The problem in Wi-Fi network is overcome by the WiMAX network. Comparing these, Wi-Fi network and WiMAX technology is more secure and reliable service. As a result, merging these two technologies gives us better result and response with respect to quality of service, mobility, coverage and practicability.

So it can be concluded that merging of two technologies results in better delivery of QoS services especially in case of voice with data.

To generate a optimized and effective routing protocol can be one of the scope for future work.

For future work it can be elaborate and explore the heterogeneous network with different models and routing protocol.

\section{ACKNOWLEDGEMENT}

I express profound sense of gratitude to my advisor Dr. Rajeev Paulus, Department of Electronics \& Communication Engineering, Sam Higginbottom Institute of Agriculture, Technology and Sciences, Deemed University, Naini, Allahabad, for his motivation and giving me adequate of time to educate me. I appreciate Professor Rajeev Paulus for his continuous help and support. Also, I would like to thank all the staff of the department for their constant support and help all the way through.

\section{REFERENCES}

[1] A.Pontes, D.dos Passos Silva, J.Jailton, O.Rodrigues, and K.L.Dias, (October 2008) "Handover management in integrated WLAN and mobile WiMAX networks," In IEEE Wireless Communications, Volume 15, Issue 5,pp.86-95.

[2] E.Gustafsson and A.Jonsson, (Feb 2003) "Always best connected," In IEEE Wireless Communications, Volume 10, Issue 1, pp.49-55.

[3] K.Kong , W.Lee, Y.Han, M.Shin, and H. You, (April 2008) "Mobility management for ALL-IP Mobile Networks: Mobile IPv6 vs. Proxy Mobile IPv6." In IEEE Wireless Communications, Volume 15, Issue 2, pp 36-45.

[4] K.Anderson, (November 2010) "On Access Network Selection Models and Mobility Support in Heterogeneous networks," Doctoral Thesis, Mobile System, Department of Computer Science and Electrical Engineering, Lulea University of Technology, SE-97-187Lulea, Sweden.

[5] M.Shekhar, K.Kumar, S.Kumar, (July 2012) "Global Mobility: The Key Enable for Next Generation Networks (NGN)," Int J. of Information \& Electronics Engineering vol. 2, no. 4.

[6] M.Suresh, G.Jabert, C.Kamalanathan, S. Kirubakaran, S.Valarmathy, (2013) "Improving the Performance of WIMAX Using Various Routing Protocols,"IJARCSSE, vol. 3, pp.230-233.

[7] V.Malhotra, L.Kumar(July 2013) "Qos Based Analysis in IEEE802.11 and IEEE 802.16 Integrated Networks, "IJARCSSE,vol. 3,issue 7,pp.1108-1113

[8] QualNet documentation," Qualnet 6.1 Model Library. 